\title{
STRUCTURAL MODEL OF ABILITY DISTRIBUTION IN THE ITEM RESPONSE THEORY
}

\author{
Haruhiko Ogasawara*
}

\begin{abstract}
A structural model of the ability distribution in the item response theory is proposed in which continuous covariates representing the properties of groups to which the subjects belong are involved. The ability parameter which is treated as a random variable, is regressed on the covariates. It is assumed that the ability parameter is normally distributed with the mean and variance which are functions of the covariates. A two-parameter logistic model is adopted as the probability model of an item response. It is shown that item parameters and hyperparameters in the ability distribution can be estimated simultaneously using the EM algorithm. Examples are provided, where the mean and standard deviation of the ability parameter are represented by polynomials of age for data of an adult intelligence test.
\end{abstract}

\section{Introduction}

In the item response theory, parameters for items and parameters for abilities are involved. Assuming that any parameters have fixed values, although they are unknown, we have as many ability parameters as the number of subjects. It is well known that even if the number of subjects is increased the estimates of parameters do not approach true values, that is, the maximum likelihood estimators have not consistency.

To avoid this situation the ability parameters are supposed to be random variables and are integrated out from the likelihood (Bock \& Lieberman, 1970; Bock \& Aitkin, 1981; Tsutakawa, 1984). This procedure is called marginal maximum likelihood estimation, in which, for instance, normal distributions and non-parametric distributions with finite values are used for the distributions of ability parameters (e.g. Bock \& Aitkin, 1981).

We consider the following 2-parameter logistic model :

$$
P_{i j}=1 /\left(1+\exp \left(-a_{j}\left(\theta_{i}-b_{j}\right)\right)\right.
$$

where $a_{j}$ and $b_{j}$ are the item parameters for the $j$-th item, and $\theta_{i}$ is the ability parameter for the $i$-th individual. The equation (1) represents the probability of correct response of the $i$-th individual to the $j$-th item. From (1) we see that there exists indeterminacy with respect to origin and scale for item parameters or ability parameters. However, when the item parameters are known from preceding experiments or when the item parameters can be estimated independently of ability parameters in models such as Rasch model, estimation of the mean and variance of

Key Words and Phrases; item response theory, logistic model, structural model, ability parameter, EM algorithm

* Railway Technical Research Institute, Hikari-cho, Kokubunji, Tokyo, 185 Japan. 
ability parameters is meaningful (Andersen \& Madsen, 1977; Andersen, 1980; Mislevy, 1984 ; Rigdon \& Tsutakawa, 1983; Sanathanan \& Blumenthal, 1978).

When data consist of several groups, where different distributions of ability parameters are assumed, the contrast of ability-distribution parameters among the groups may be estimated (Mislevy, 1985). The papers mentioned above contain the procedure of testing the differences of means and/or variances of $\theta_{i}$. The test of means and variances of $\theta_{i}$ in the item response theory is similar to that of the factor analysis model for several groups (Jöreskog, 1971 ; Sörbom, 1974) in that only the contrast may be tested.

In the item response theory for several groups, if we employ dummy variables which represent the membership of individuals to groups, the model can be regarded as a regression model of ability parameters on the dummy variables. Further, we can use continuous covariates such as age and income. Reiser (1983) introduced group effects as fixed parameters in the item response model. Mislevy (1987) proposed a homoscedastic linear regression model of $\theta_{i}$ on auxiliary variables. However, in the former model an individual responds to only one item. In the latter model $\theta_{i}$ 's are estimated in the first stage, then the estimated $\theta_{i}$ 's are regressed on the auxiliary variables by usual least squares.

In this paper, when continuous covariates for individuals are available and when the distributions of ability parameters are described by parameters which are structured with the covariates, the method of estimating the structural parameters will be described without estimating individual ability parameters. The above regression model will be extended to the case of unequal variance of ability distribution. These extensions correspond to those for the factor analysis model in which latent factors are regressed on observed continuous covariates (Ogasawara, 1990).

\section{Models}

Let $\boldsymbol{x}_{i}$ denote the vector of the responses of the $i$-th individual $(i=1, \ldots, N)$ for $p$ items, where $N$ is the number of individuals:

$$
\boldsymbol{x}_{i}=\left(x_{i 1}, \ldots, x_{i p}\right)^{\prime}, i=1, \ldots, N
$$

in which $x_{i j}$ is the response of the $i$-th individual to the $j$-th item. $x_{i j}=1$ stands for correct response and $x_{i j}=0$ otherwise. Let $P_{i j}$ denote the probability of $x_{i j}=1$. We assume that $P_{i j}$ is described by the following logistic function which is essentially the same as (1):

$$
P_{i j}=\frac{1}{1+\exp \left(-\left(\beta_{1 j}+\beta_{2 j} \theta_{i}\right)\right)},
$$

where $\beta_{1 j}$ and $\beta_{2 j}$ are the item parameters for the $j$-th item $\left(\beta_{j}=\left(\beta_{1 j}, \beta_{2 j}\right)^{\prime}\right)$ and $\theta_{i}$ is the ability parameter for the $i$-th individual. Then, the probability function of 
$\boldsymbol{x}_{i}$ given $\theta_{i}$ and $\beta_{j}, j=1, \ldots, p$ is obtained with the assumption of local independence :

$$
P\left(\boldsymbol{x}_{i} \mid \theta_{i}, \boldsymbol{\beta}\right)=\prod_{j=1}^{p} P_{i j}^{x_{i j}} Q_{i j}^{1-x_{i j}},
$$

where $\beta=\left(\beta_{1}{ }^{\prime}, \ldots, \beta_{p}{ }^{\prime}\right)^{\prime}$ and $Q_{i j}=1-P_{i j}$. The vector $\beta$ consists of fixed and unknown parameters. The parameters, $\theta_{i}, i=1, \ldots, N$, are assumed to be independent random variables which are distributed according to possibly unidentical normal distributions:

$$
\theta_{i} \sim N\left(\mu\left(\boldsymbol{t}_{i}, \boldsymbol{\gamma}\right), \sigma^{2}\left(\boldsymbol{t}_{i}, \boldsymbol{\delta}\right)\right), i=1, \ldots, N,
$$

where $\mu\left(\boldsymbol{t}_{i}, \boldsymbol{\gamma}\right)$ is the mean of $\theta_{i}$ and denotes that it is a function of $\boldsymbol{t}_{i}\left(=\left(t_{i 1}, \ldots, t_{i w}\right)^{\prime}\right)$, the vector of $w$ covariates with the hyperparameter $\boldsymbol{\gamma}$. Similarly, $\sigma^{2}\left(\boldsymbol{t}_{\boldsymbol{i}}, \boldsymbol{\delta}\right)$ represents the variance of $\theta_{i}$, which is a function of $t_{i}$ with the hyperparameter $\delta$. The probability density of $\theta_{i}$ is described as:

$$
\phi_{i}\left(\theta_{i} \mid \boldsymbol{\gamma}, \boldsymbol{\delta}\right)=\frac{1}{\sqrt{2 \pi} \sigma_{i}} \exp \left(-\frac{\left(\theta_{i}-\mu_{i}\right)^{2}}{2 \sigma_{i}^{2}}\right),
$$

where $\mu_{i}$ and $\sigma_{i}$ are abbreviations of $\mu\left(\boldsymbol{t}_{i}, \boldsymbol{\gamma}\right)$ and $\sigma\left(\boldsymbol{t}_{i}, \boldsymbol{\delta}\right)$, respectively. Though in (6) actual forms of $\mu_{i}$ and $\sigma_{i}$ are not given, linear models, for example, may be described as $\mu_{i}=\boldsymbol{t}_{i}^{\prime} \boldsymbol{\gamma}$ and $\sigma_{i}=\boldsymbol{t}_{\boldsymbol{i}}^{\prime} \boldsymbol{\delta}$. The parameters $\theta_{i}, i=1, \ldots, N$, are not observed and if they are of no interest, the marginal likelihood of the item parameters $\beta$ and the structural parameters (hyperparameters), $\boldsymbol{\gamma}$ and $\boldsymbol{\delta}$, given $X\left(=\left(\boldsymbol{x}_{1}, \ldots, \boldsymbol{x}_{\boldsymbol{N}}\right)^{\prime}\right)$ and $T\left(=\left(\boldsymbol{t}_{i}, \ldots, \boldsymbol{t}_{N}\right)^{\prime}\right)$ is obtained by integrating out $\theta_{i}, i=1, \ldots, N$ :

$$
L(\boldsymbol{\beta}, \boldsymbol{\gamma}, \boldsymbol{\delta} \mid X, T)=\prod_{i=1}^{N} \int_{-\infty}^{\infty} P\left(\boldsymbol{x}_{i} \mid \theta_{i}, \boldsymbol{\beta}\right) \phi_{i}\left(\theta_{i} \mid \boldsymbol{\gamma}, \boldsymbol{\delta}\right) d \theta_{i}
$$

Since it is difficult to obtain the integration algebraically, we use the Gaussian quadrature (Bock \& Aitkin, 1981; Harwell et al., 1988) with appropriate $K$ points, $\varphi_{i q}, q=1, \ldots, K$, in the distribution of $\theta_{i}$. Using this, (7) can be approximated by

$$
L \cong \prod_{i=1}^{N} \sum_{q=1}^{K} P\left(\boldsymbol{x}_{i} \mid \varphi_{i q}, \boldsymbol{\beta}\right) \frac{\phi_{i}\left(\varphi_{i q} \mid \boldsymbol{\gamma}, \boldsymbol{\delta}\right)}{\sum_{r=1}^{K} \phi_{i}\left(\varphi_{i r} \mid \boldsymbol{\gamma}, \boldsymbol{\delta}\right)}
$$

If we treat $\varphi_{i q}$ as random variables, we can write:

$$
\varphi_{i q} \sim N\left(\mu_{i}, \sigma_{i}{ }^{2}\right), q=1, \ldots, K \text {. }
$$

Let

$$
w_{q}=\frac{\phi_{i}\left(\varphi_{i q} \mid \boldsymbol{\gamma}, \boldsymbol{\delta}\right)}{\sum_{r=1}^{K} \phi_{i}\left(\varphi_{i r} \mid \boldsymbol{\gamma}, \boldsymbol{\delta}\right)}
$$

Then, $\sum_{q=1}^{K} w_{q}=1$ and we can construct $w_{q}(q=1, \ldots, K)$ which do not depend on $i$. Let

$$
\varphi_{i q}=\sigma_{i} \xi_{q}+\mu_{i}
$$


then $\xi_{q}, q=1, \ldots, K$, can be written similarly to (9) as

$$
\xi_{q}, \sim N(0,1) q=1, \ldots, K \text {. }
$$

For constructing $\varphi_{i q}$, we can choose appropriate $K$ points $\xi_{q}, q=1, \ldots, K$ from the standardized normal distribution and substitute them in (11).

\section{Estimation of parameters : Method I}

The maximum likelihood estimate of $\beta$ is obtained by maximizing the logarithm of (8) as an approximation of (7) in the following way using the EM algorithm (Dempster et al., 1977 ; Harwell et al., 1988). The partial derivative of $\ln L$ with respect to $\boldsymbol{\beta}_{j}$ is

$$
\frac{\partial \ln L}{\partial \boldsymbol{\beta}_{j}} \cong \sum_{i=1}^{N} \sum_{q=1}^{K} \frac{\partial \ln P\left(\boldsymbol{x}_{i} \mid \varphi_{i q}, \boldsymbol{\beta}\right)}{\partial \boldsymbol{\beta}_{j}} \frac{P\left(\boldsymbol{x}_{i} \mid \varphi_{i q}, \boldsymbol{\beta}\right) w_{q}}{\sum_{r=1}^{K} P\left(\boldsymbol{x}_{i} \mid \varphi_{i r}, \boldsymbol{\beta}\right) w_{r}} .
$$

Let

$$
f\left(\varphi_{i q} \mid \boldsymbol{x}_{i}, \boldsymbol{\beta}\right)=\frac{P\left(\boldsymbol{x}_{i} \mid \varphi_{i q}, \boldsymbol{\beta}\right) w_{q}}{\sum_{i=1}^{K} P\left(\boldsymbol{x}_{i} \mid \varphi_{i r}, \boldsymbol{\beta}\right) w_{r}}
$$

then $f\left(\varphi_{i q} \mid \boldsymbol{x}_{i}, \boldsymbol{\beta}\right)$ is the probability density function of the posterior distribution of $\varphi_{i q}$ given $\boldsymbol{x}_{i}$ and $\boldsymbol{\beta}$, and (13) becomes

$$
\begin{aligned}
\frac{\partial \ln L}{\partial \boldsymbol{\beta}_{j}} & \cong \sum_{i=1}^{N} \sum_{q=1}^{K} \frac{\left(x_{i j}-P_{q j}\right)}{P_{q j} Q_{q j}} \frac{\partial P_{q j}}{\partial \boldsymbol{\beta}_{i}} f\left(\varphi_{i q} \mid \boldsymbol{x}_{i}, \boldsymbol{\beta}\right) \\
& =\sum_{q=1}^{K} \sum_{i=1}^{N}\left[\begin{array}{c}
\left(x_{i j}-P_{q j}\right) \\
\varphi_{i q}\left(x_{i j}-P_{q j}\right)
\end{array}\right] f\left(\varphi_{i q} \mid \boldsymbol{x}_{i}, \boldsymbol{\beta}\right),
\end{aligned}
$$

where $\sum_{i=1}^{N} x_{i j} f\left(\varphi_{i q} \mid \boldsymbol{x}_{i}, \boldsymbol{\beta}\right)$ and $\sum_{i=1}^{K} f\left(\varphi_{i q} \mid \boldsymbol{x}_{i}, \boldsymbol{\beta}\right)$ correspond to the number of responses of interest and the number of subjects at the $q$-th level of stimulus, respectively, in the ordinary stimulus-response model (see e.g. Bock \& Jones, 1980). In the context of the EM algorithm they are called "expected frequency" and "expected sample size" (Bock \& Aitkin, 1981). Using provisional values of $\boldsymbol{\beta}, \boldsymbol{\gamma}$ and $\boldsymbol{\delta}$. We treat $f\left(\boldsymbol{\varphi}_{i q} \mid \boldsymbol{x}_{\boldsymbol{i}}\right.$, $\beta$ ) as constant valuwes, althouth they are actually functions of $\beta, \gamma$ and $\delta$. Then, we have simple expressions of the expectations of the second partial derivatives:

$$
\begin{aligned}
& E\left(\frac{\partial^{2} \ln L}{\partial \boldsymbol{\beta}_{j} \partial \boldsymbol{\beta}_{j}^{\prime}}\right) \cong-\sum_{q=1}^{K} \sum_{i=1}^{N}\left[\begin{array}{cc}
1 & \varphi_{i q} \\
\varphi_{i q} & \varphi^{2}{ }_{i q}
\end{array}\right] P_{q j} Q_{q j} f\left(\varphi_{i q} \mid \boldsymbol{x}_{i}, \boldsymbol{\beta}\right), \\
& E\left(\frac{\partial^{2} \ln L}{\partial \boldsymbol{\beta}_{j} \partial \boldsymbol{\beta}_{k}^{\prime}}\right)=0 . \\
& \quad(j \neq k)
\end{aligned}
$$

Using these expressions, the revised value of $\beta$ is obtained iteratively by Fisher's scoring method which is used in ordinary stimulus-response models in the following way: 


$$
\boldsymbol{\beta}_{(s+1)}=\boldsymbol{\beta}_{(s)}-\left\{\left.E\left(\frac{\partial^{2} \ln L}{\partial \beta \partial \beta^{\prime}}\right)\right|_{\beta=\beta_{s s}}\right\}^{-1},\left.\frac{\partial \ln L}{\partial \beta}\right|_{\beta=\beta_{s s},}
$$

where $\beta_{(s)}$ is the value of the vector $\beta$ in the $s^{- \text {th }}$ iteration.

Similarly, the value of $\boldsymbol{\gamma}$ and $\delta$ are revised iteratively in the following manner. The partial derivative of $\ln L$ with respect to $\gamma$ is

$$
\begin{aligned}
\frac{\partial \ln L}{\partial \boldsymbol{\gamma}} & =\sum_{i=1}^{N} \frac{\int_{-\infty}^{\infty} P\left(\boldsymbol{x}_{i} \mid \theta_{i}, \boldsymbol{\beta}\right)\left(\partial \phi_{i}\left(\theta_{i} \mid \boldsymbol{\gamma}, \boldsymbol{\delta}\right) / \partial \boldsymbol{\gamma}\right) d \theta_{i}}{\int_{-\infty}^{\infty} P\left(\boldsymbol{x}_{i} \mid \theta_{i}, \boldsymbol{\beta}\right) \phi_{i}\left(\theta_{i} \mid \boldsymbol{\gamma}, \boldsymbol{\delta}\right) d \theta_{i}} \\
& =\sum_{i=1}^{N} \frac{\int_{-\infty}^{\infty}\left(\boldsymbol{x}_{\boldsymbol{i}} \mid \theta_{i}, \boldsymbol{\beta}\right) \phi_{i}\left(\theta_{i} \mid \boldsymbol{\gamma}, \boldsymbol{\delta}\right)}{\int_{-\infty}^{\infty} P\left(\boldsymbol{x}_{i} \mid \theta_{r}, \boldsymbol{\beta}\right) \phi_{r}\left(\theta_{r} \mid \boldsymbol{\gamma}, \boldsymbol{\delta}\right) d \theta_{r}} \frac{\left(\theta_{i}-\mu_{i}\right)}{\sigma_{i}{ }^{2}} \frac{\partial \mu_{i}}{\partial \boldsymbol{\gamma}} d \theta_{i} \\
& \cong \sum_{q=1}^{K} \sum_{i=1}^{N} f\left(\varphi_{i q} \mid x_{i}, \boldsymbol{\beta}\right) \frac{\left(\varphi_{i q}-\mu_{i}\right)}{\sigma_{i}{ }^{2}} \frac{\partial \mu_{i}}{\partial \boldsymbol{\gamma}}
\end{aligned}
$$

where $f\left(\varphi_{i q} \mid \boldsymbol{x}_{i}, \boldsymbol{\beta}\right)$ is the posterior distribution of $\varphi_{i q}$ mentioned above, and is a function of $\boldsymbol{\gamma}$ and $\delta$ as well as $\beta$, because $\varphi_{i q}=\sigma_{i} w_{q}+\mu_{i}$. Similarly,

$$
\frac{\partial \ln L}{\partial \boldsymbol{\delta}} \cong \sum_{q=1}^{K} \sum_{i=1}^{N} f\left(\varphi_{i q} \mid \boldsymbol{x}_{i}, \beta\right)\left\{-\frac{1}{\sigma_{i}}+\frac{\left(\varphi_{i q}-\mu_{i}\right)^{2}}{\sigma_{i}{ }^{2}}\right\} \frac{\partial \sigma_{i}}{\partial \boldsymbol{\delta}}
$$

is obtained. Suppose that $f\left(\varphi_{i q} \mid x_{i}, \beta\right)$ are given, then

$$
\begin{aligned}
& E\left(\frac{\partial^{2} \ln L}{\partial \boldsymbol{\gamma} \partial \boldsymbol{\gamma}^{\prime}}\right) \cong-\sum_{q=1}^{K} \sum_{i=1}^{N} f\left(\varphi_{i q} \mid \boldsymbol{x}_{i}, \boldsymbol{\beta}\right) \frac{1}{\sigma_{i}{ }^{2}} \frac{\partial \mu_{i}}{\partial \boldsymbol{\gamma}} \frac{\partial \mu_{i}}{\partial \boldsymbol{\gamma}^{\prime}}, \\
& E\left(\frac{\partial^{2} \ln L}{\partial \boldsymbol{\gamma} \partial \boldsymbol{\delta}^{\prime}}\right)=0, \\
& E\left(\frac{\partial^{2} \ln L}{\partial \boldsymbol{\delta} \partial \boldsymbol{\delta}^{\prime}}\right) \cong-\sum_{q=1}^{K} \sum_{i=1}^{N} f\left(\varphi_{i q} \mid \boldsymbol{x}_{i}, \boldsymbol{\beta}\right) \frac{2}{\sigma_{i}{ }^{2}} \frac{\partial \sigma_{i}}{\partial \boldsymbol{\delta}} \frac{\partial \sigma_{i}}{\partial \boldsymbol{\delta}^{\prime}} .
\end{aligned}
$$

The equations (16), (17) and (18) are similar to the expressions for the models of $\mu_{i}$ and $\sigma_{i}$ in which $\theta_{i}$ are observed values (Ogasawara, 1986), though they are weighted by $f\left(\varphi_{i q} \mid \boldsymbol{x}_{i}, \boldsymbol{\beta}\right)$ in our model. We can improve the values of $\boldsymbol{\gamma}$ and $\boldsymbol{\delta}$ by Fisher's scoring method using (16), (17) and (18).

After replacing $\gamma$ and $\delta$ with their improved values and fixing these values, we again improve the value of $\beta$. This alternate cycle may be repeated until estimated values of the parameters become unchanged.

\section{Estimation of parameters : Method II}

It is possible to obtain estimates of the parameters by Method I. However, the speed of convergence is rather slow when the structure of $\mu_{i}$ and $\sigma_{i}$ become complicated, although the expressions of (14) and (18) are very simple. In this section we propose the second procedure (Method II) of estimating the parameters. Method II employs a little different model but we can show that it also fits data just as well as the model in Method I does. 
The ability parameter $\theta_{i}$ can be written as $\theta_{i}=\sigma_{i} \xi+\mu_{i}$ by introducing a new random variable $\xi$. Using this reparameterization, (7) becomes

$$
L=\prod_{i=1}^{N} \int_{-\infty}^{\infty} P\left(\boldsymbol{x}_{i} \mid \sigma_{i} \xi+\mu_{i}, \boldsymbol{\beta}\right) \phi(\xi) d \xi,
$$

where

$$
\phi(\xi)=\frac{1}{\sqrt{2 \pi}} \exp \left(-\frac{\xi^{2}}{2}\right) .
$$

Adopting finite typical points, $\xi_{q}, q=1, \ldots, K$, in the distribution of $\xi$, the integration in (19) is approximated by

$$
L \cong \prod_{i=1}^{N} \sum_{q=1}^{K} P\left(\boldsymbol{x}_{i} \mid \sigma_{i} \xi+\mu_{i}, \beta\right) \frac{\phi\left(\xi_{q}\right)}{\sum_{r=1}^{K} \phi\left(\xi_{r}\right)} .
$$

Assume that $\xi_{q}$ in (12) are identical to $\xi_{q}$ in (21) without loss of generality, then cosidering the relationship $\varphi_{i q}=\sigma_{i} \xi_{q}+\mu_{i}$ we easily obtain

$$
w_{q}=\frac{\phi_{i}\left(\varphi_{i q} \mid \boldsymbol{\gamma}, \boldsymbol{\delta}\right)}{\sum_{q=1}^{K} \phi_{i}\left(\varphi_{i r} \mid \boldsymbol{\gamma}, \boldsymbol{\delta}\right)}=\frac{\phi\left(\xi_{q}\right)}{\sum_{r=1}^{K} \phi\left(\xi_{r}\right)} .
$$

We see that (19) is always equal to (7) and that (21) is equal to (8) if $\varphi_{\text {iq }}$ are constructed by (11). Hence we have the same estimates of the parameters by maximizing the value of (21) as those obtained by Method I. The difference between Methods I and II in the computations is that in Method I $\varphi_{i q}$ are treated as fixed values when $\beta$ are estimated, while in Method II $\xi_{q}$ are treated as fixed values. Since $\xi$ is normally distributed with zero mean and unit variance, $\xi$ is interpreted as a variable which represents the standardized ability given $\boldsymbol{t}_{i}$.

Improvement of the values of $\beta, \gamma$ and $\delta$ is performed simultaneously for these parameters instead of using the alternate procedure which was adopted by Method I. Hereafter, we write $P\left(\boldsymbol{x}_{i} \mid \sigma_{i} \xi+\mu_{i}, \boldsymbol{\beta}\right)$ as $P\left(\boldsymbol{x}_{i} \mid \xi, \boldsymbol{\beta}, \boldsymbol{\gamma}, \boldsymbol{\delta}\right)$. Then we have the partial derivatives:

$$
\begin{aligned}
\frac{\partial \ln L}{\partial \boldsymbol{\beta}_{j}} & \cong \sum_{i=1}^{N} \sum_{q=1}^{K} \frac{\partial \ln P\left(\boldsymbol{x}_{i} \mid \xi_{q}, \boldsymbol{\beta}, \boldsymbol{\gamma}, \boldsymbol{\delta}\right)}{\partial \boldsymbol{\beta}_{j}} \frac{P\left(\boldsymbol{x}_{\boldsymbol{i}} \mid \xi_{q}, \boldsymbol{\beta}, \boldsymbol{\gamma}, \boldsymbol{\delta}\right) w_{q}}{\sum_{r=1}^{K} P\left(\boldsymbol{x}_{i} \mid \xi_{r}, \boldsymbol{\beta}, \boldsymbol{\gamma}, \boldsymbol{\delta}\right) w_{r}} \\
& =\sum_{i=1}^{N} \sum_{q=1}^{K} \frac{\partial \ln P\left(\boldsymbol{x}_{i} \mid \xi_{q}, \boldsymbol{\beta}, \boldsymbol{\gamma}, \boldsymbol{\delta}\right)}{\partial \boldsymbol{\beta}_{j}} f\left(\xi_{q} \mid \boldsymbol{x}_{i}, \boldsymbol{\beta}, \boldsymbol{\gamma}, \boldsymbol{\delta}\right) \\
& =\sum_{q=1}^{K} \sum_{i=1}^{N}\left(\begin{array}{c}
x_{i j}-P_{q j} \\
\left(\sigma_{i} \xi_{q}+\mu_{i}\right)\left(x_{i j}-P_{q j}\right)
\end{array}\right) f\left(\xi_{q} \mid \boldsymbol{x}_{i}, \boldsymbol{\beta}, \boldsymbol{\gamma}, \boldsymbol{\delta}\right)
\end{aligned}
$$

where $f\left(\xi_{q} \mid \boldsymbol{x}_{i}, \boldsymbol{\beta}, \boldsymbol{\gamma}, \boldsymbol{\delta}\right)$ is the probability density function of the posterior distribution of $\xi_{q}$ given $\boldsymbol{x}_{\boldsymbol{i}}$ and the values of the parameters. This density function is identical to $f\left(\varphi_{i q} \mid \boldsymbol{x}_{i}, \boldsymbol{\beta}\right)$, because $\varphi_{i q}=\sigma_{i} \xi_{q}+\mu_{i}$.

Similarly we have 


$$
\begin{aligned}
& \frac{\partial \ln L}{\partial \boldsymbol{\gamma}} \cong \sum_{q=1}^{K} \sum_{j=1}^{P} \sum_{i=1}^{N} \beta_{2 j}\left(x_{i j}-P_{q j}\right) f\left(\xi_{q} \mid x_{i}, \boldsymbol{\beta}, \boldsymbol{\gamma}, \boldsymbol{\delta}\right) \frac{\partial \mu_{i}}{\partial \boldsymbol{\gamma}}, \\
& \frac{\partial \ln L}{\partial \boldsymbol{\delta}} \cong \sum_{q=1}^{K} \sum_{j=1}^{P} \sum_{i=1}^{N} \beta_{2 j} \xi_{q}\left(x_{i j}-P_{q j}\right) f\left(\xi_{q} \mid x_{i}, \boldsymbol{\beta}, \boldsymbol{\gamma}, \boldsymbol{\delta}\right) \frac{\partial \sigma_{i}}{\partial \boldsymbol{\delta}} .
\end{aligned}
$$

The information matrix for the scoring method with the assumption of fixed $f\left(\xi_{q} \mid \boldsymbol{x}_{i}, \boldsymbol{\beta}, \boldsymbol{\gamma}, \boldsymbol{\delta}\right)$ is provided in Appendix. For the standard errors of the estimates of $\boldsymbol{\beta}, \boldsymbol{\gamma}$ and $\boldsymbol{\delta}$, we cannot use the information matrices in Methods I and II, since they are computed as if $\theta_{i}$ were observed (Louis, 1982; Mislevy \& Sheehan, 1989). Instead we use the following approximation of the exact information matrix (Mislevy, 1984, 1985).

$$
I \cong \sum_{i=1}^{N} \frac{\partial \ln L_{i}}{\partial\left(\boldsymbol{\beta}^{\prime}, \boldsymbol{\gamma}^{\prime}, \boldsymbol{\delta}^{\prime}\right)^{\prime}} \frac{\partial \ln L_{i}}{\partial\left(\boldsymbol{\beta}^{\prime}, \boldsymbol{\gamma}^{\prime}, \boldsymbol{\delta}^{\prime}\right)}
$$

where

$$
\frac{\partial \ln L_{i}}{\partial\left(\boldsymbol{\beta}^{\prime}, \boldsymbol{\gamma}^{\prime}, \boldsymbol{\delta}^{\prime}\right)}=\sum_{q=1}^{K} \frac{\partial \ln P\left(\boldsymbol{x}_{i} \mid \xi_{q}, \boldsymbol{\beta}, \boldsymbol{\gamma}, \boldsymbol{\delta}\right)}{\partial\left(\boldsymbol{\beta}^{\prime}, \boldsymbol{\gamma}^{\prime}, \boldsymbol{\delta}^{\prime}\right)} f\left(\xi_{q} \mid \boldsymbol{x}_{i}, \boldsymbol{\beta}, \boldsymbol{\gamma}, \boldsymbol{\delta}\right) .
$$

\section{Examples}

Two data sets of an adult intelligence test are used as examples of our model. A non-verbal intelligence test, Test A (Ogasawara, 1986), consists of four subtests $\mathrm{A} 1, \mathrm{~A} 2, \mathrm{~A} 3$ and $\mathrm{A} 4$, where $\mathrm{A} 1$ and $\mathrm{A} 2$ are subtests to measure mainly perceptual ability and $\mathrm{A} 3$ and $\mathrm{A} 4$ are subtests to measure reasoning and spatial ability. The four subtests were administered to 1,495 male adults aged from 20 to 54 years old. The original raw scores of Test A were the number-right scores of the subtests. The average score of each subtest in an age group tends to decrease with age in our data. That is, age is a covariate in the distributions of the scores. The decreasing tendency of the scores in $\mathrm{A} 1$ and $\mathrm{A} 2$ seems to be quadratic in age, which is convex upward, while the tendency of A3 and A4 is linear in age. The variance of the scores given an age seems to be constant or increasing with age for $\mathrm{Al}$ and $\mathrm{A} 2$, and decreasing for A3 and A4 (Ogasawara, 1986).

Here, we treat the subtests as items with binary responses in the following way. If the score of the $j$-th subtest for the $i$-th individual is greater than a certain value, $x_{i j}=1$ (the case of success). If the score is less than or equal to the value, $x_{i j}=0$ (the case of failure). Considering the contents of the subtests, the data of the subtests were divided into two data sets (A1, A2) and (A3, A4) to be analyzed separately.

Table 1 shows the proportions of success $\left(x_{i j}=1\right)$ in age groups. From Table 1 it is seen that the proportions of success in each subtest decreases with age, which should reflect the difference of distributions of latent ability. Thus, we assume that $\mu_{i}$ and $\sigma_{i}$ are described by polynomials of appropriate orders with respect to age $\left(t_{i}\right)$ : 
Table 1

Proportions of success in age groups

\begin{tabular}{ll|ll|ll}
\hline \multirow{2}{*}{$\begin{array}{c}\text { Age group } \\
\text { (years) }\end{array}$} & $N$ & \multicolumn{4}{c}{ Subtest } \\
\cline { 3 - 6 } & A1 & A2 & A3 & A4 \\
\hline $20 \sim 24$ & 219 & .566 & .731 & .763 & .776 \\
$25 \sim 29$ & 276 & .634 & .764 & .703 & .764 \\
$30 \sim 34$ & 142 & .570 & .718 & .634 & .613 \\
$35 \sim 39$ & 265 & .517 & .592 & .483 & .483 \\
$40 \sim 44$ & 287 & .477 & .429 & .422 & .429 \\
$45 \sim 49$ & 189 & .280 & .312 & .286 & .307 \\
$50 \sim 54$ & 117 & .120 & .179 & .154 & .205 \\
\hline
\end{tabular}

$$
\begin{aligned}
& \mu_{i}=\gamma_{1} t_{i}+\gamma_{2} t_{i}{ }^{2}+\cdots+\gamma_{u} t_{i}{ }^{u} \\
& \sigma_{i}=1+\delta_{1} t_{i}+\delta_{2} t_{i}{ }^{2}+\cdots+\delta_{v} t_{i}{ }^{v}
\end{aligned}
$$

where $\mu_{i}$ and $\sigma_{i}$ are set at zero and one, respectively, for $t_{i}=0$ considering the arbitrariness of the origin and scale of $\theta_{i}$ mentioned previously. From (27)

$$
\boldsymbol{\gamma}=\left(\gamma_{1}, \cdots, \gamma_{u}\right)^{\prime}, \boldsymbol{\delta}=\left(\delta_{1}, \cdots, \delta_{v}\right)^{\prime}
$$

All the results were obtained by Method II except that several simple models were also obtained by Method I. Typical ten points in the standardized normal distribution ( $\pm .444, \pm 1.333, \pm 2.222, \pm 3.111, \pm 4.000$; Harwell et al., 1988) with their appropriate weights were used for the Gaussian quadrature. In Tables $2 \sim 4$ are shown the results of fitting the models to the data set of A1 and A2. The orders of polynomials fitted for $\mu_{i}$ and $\sigma_{i}$ are $1 \sim 3$ and $0 \sim 1$, respectively. In the case of the order of two for $\sigma_{i}$ convergence was not obtained within ordinary computing time. Table 2 shows the AIC's (Akaike information criterion; Akaike, 1973) and the $t$ values (the estimated value of a parameter/its estimated standard error) for the coefficients of the highest power terms in the polynomials of the minimum AIC model. Table 3 shows the estimates of the parameters and their standard errors. In Table 4 are given estimated probabilities of the minimum AIC model at median

Table 2

Results for Subtests A1 and A2

\begin{tabular}{c|rrc|rrrr}
\hline $\begin{array}{c}\text { Order } \\
\text { for } \sigma\end{array}$ & \multicolumn{3}{|c|}{0} & \multicolumn{3}{|c}{1} \\
\hline $\begin{array}{c}\text { Order } \\
\text { for } \mu\end{array}$ & AIC & $t$ for $\mu$ & $t$ for $\sigma$ & AIC & $t$ for $\mu$ & $t$ for $\sigma$ \\
\hline 1 & 3740.00 & -11.56 & -- & 3739.41 & -4.76 & -2.36 \\
2 & 3706.67 & -5.70 & -- & 3704.24 & -0.60 & 0.49 \\
3 & 3706.79 & 1.34 & -- & 3705.10 & 0.70 & 0.57 \\
\hline
\end{tabular}


Table 3

Estimates of the parameters in the minimum AIC model for Subtests A1 and A2

\begin{tabular}{c|c|cc}
\hline Subtest A1 & $\beta_{1}:-2.551(.844)$ & $\beta_{2}: .2332(.3662)$ \\
Subtest A2 & $\beta_{1}:-3.878(1.430)$ & $\beta_{2}: .3987(.6373)$ \\
\hline$\mu:$ & $.9864 t-.01827 t^{2}$ & $\sigma:$ & $1+.09082 t$ \\
& $(.03037)$ & & $(.18608)$ \\
\hline
\end{tabular}

Note. $t=$ Age (years). The numbers in parentheses indicate standard errors.

Table 4

Estimated probabilities and estimated $\mu_{i}$ and $\sigma_{i}$ at various ages in the minimum AIC model for Subtests A1 and A2

\begin{tabular}{c|cc|cc}
\hline Age (years) & \multicolumn{2}{|c|}{ Probability } & \multicolumn{2}{c}{} \\
& $\mathrm{A} 1$ & $\mathrm{~A} 2$ & $\mu_{i}$ & $\sigma_{i}$ \\
\hline 22 & .610 & .777 & 12.86 & 3.00 \\
27 & .635 & .807 & 13.31 & 3.45 \\
32 & .610 & .777 & 12.85 & 3.91 \\
37 & .532 & .668 & 11.48 & 4.36 \\
42 & .400 & .447 & 9.19 & 4.81 \\
47 & .240 & .184 & 5.99 & 5.27 \\
52 & .108 & .042 & 1.88 & 5.73 \\
\hline
\end{tabular}

ages of the age groups.

The orders of the polynomials of the minimum AIC model among models fitted are two and one for $\mu_{i}$ and $\sigma_{i}$, respectively. The absolute $t$-values are not large enough. However, the quadratic tendency (order of two) of $\mu_{i}$ with age (Table 4) corresponds to the observed tendency in Table 1, which implies a development in the age of twenties (increase of probability) and decay afterwards (decrease of probability). It is seen that the estimated probabilities reproduce fairly well the proportions of success in Table 1.

The order of one (linear) for $\sigma_{i}$ in the minimum AIC model corresponds to the tendency of the model for the number-right scores, in which the orders of the minimum AIC model were zero (constant) and one (linear) for A1 and A2, respectively (Ogasawara, 1986).

Tables $5 \sim 7$ give the results of fitting our model to the second data set (Subtests A3 and A4). The orders of polynomials fitted are the same as those for the first data set. The minimum AIC model in Table 5 is the model of polynomials of order of one both for $\mu_{i}$ and $\sigma_{i}$. The absolute $t$-values are large enough to support the orders of the polynomials. Table 6 shows the estimates of the parameters and their standard errors. In Table 7 the results of fitting the model are shown. The estimated probabilities seem to reproduce the observed ones as a whole, although the model overestimates the probability at around 22 years old. The decreasing tendency of $\sigma_{i}$ with age corresponds to a similar one observed in the model of $\sigma_{i}$ for 
Table 5

Results for Subtests A3 and A4

\begin{tabular}{c|rrr|crr}
\hline $\begin{array}{c}\text { Order } \\
\text { for } \sigma\end{array}$ & \multicolumn{3}{|c|}{0} & \multicolumn{3}{|c}{1} \\
\hline $\begin{array}{c}\text { Order } \\
\text { for } \mu\end{array}$ & AIC & $t$ for $\mu$ & $t$ for $\sigma$ & AIC & $t$ for $\mu$ & $t$ for $\sigma$ \\
\hline 1 & 3617.74 & -12.87 & -- & 3617.43 & -4.43 & -2.25 \\
2 & 3618.25 & -1.22 & -- & 3619.17 & -.47 & -1.44 \\
3 & 3620.18 & .26 & - & 3621.14 & .19 & -1.41 \\
\hline
\end{tabular}

Table 6

Estimates of the parameters in the minimum AIC model for Subtests $\mathrm{A} 3$ and $\mathrm{A} 4$

\begin{tabular}{|c|c|c|}
\hline $\begin{array}{l}\text { Subtests A3 } \\
\text { Subtests A4 }\end{array}$ & $\begin{array}{l}\beta_{1}: 4.503(.441) \\
\beta_{1}: 4.451(.431)\end{array}$ & $\begin{array}{l}\beta_{2}: 1.944(.461) \\
\beta_{2}: 1.871(.454)\end{array}$ \\
\hline$\mu:$ & $\begin{array}{c}-.06240 t \\
(.01408)\end{array}$ & $\begin{array}{r}1-.009041 t \\
(.004026)\end{array}$ \\
\hline
\end{tabular}

Note. $t=$ Age (years). The numbers in parentheses indicate standard errors.

Table 7

Estimated probabilities and estimated $\mu_{i}$ and $\sigma_{i}$ at various ages in the minimum AIC model for Subtests $\mathrm{A} 3$ and $\mathrm{A} 4$

\begin{tabular}{c|cc|cc}
\hline \multirow{2}{*}{ Age (years) } & \multicolumn{3}{|c|}{ Probability } & \multicolumn{2}{c}{} \\
& $\mathrm{A} 3$ & $\mathrm{~A} 4$ & $\mu_{i}$ & $\sigma_{i}$ \\
\hline 22 & .862 & .868 & -1.373 & .801 \\
27 & .774 & .786 & -1.685 & .756 \\
32 & .651 & .672 & -1.997 & .711 \\
37 & .504 & .533 & -2.309 & .665 \\
42 & .356 & .389 & -2.621 & .620 \\
47 & .232 & .262 & -2.933 & .575 \\
52 & .141 & .165 & -3.245 & .530 \\
\hline
\end{tabular}

the number-right scores for A3 and A4 (Ogasawara, 1986).

From the results of fitting our model to the two data sets, we see that there is a common tendency to our model and the model for the number-right score, which implies that the difference in the distributions of $\theta_{i}$ can be described by covariates using the information of binary responses.

\section{Discussion}

In the above example real data are used. However, the data were composed by regarding subtests as items, that is, it was supposed that a subject whose number-right score of a subtest which is greater than a criterion gives a correct 
answer to the item (actually subtest). Therefore, though there is loss of information in the transformed data, the results are meaningful when some decision is supposed to be made by judging a subject as intelligent or not intelligent by the transformed data.

An anonymous referee suggested that simulated data should be used. Then, the estimated $\mu_{i}$ and $\sigma_{i}$ can be compared with the true $\mu_{i}$ and $\sigma_{i}$. It will also be observed what relationships exist between different $\beta$. These are the tasks assigned to the author. In addition the referee pointed out that if normality is assumed for the distribution of $\theta_{i}$ for each group, then the parameters of each ability-distribution can be estimated through the equating of $N$ sets of operating characteristics of the items estimated separately for the $N$ distributions. This comment indicates a useful method when data are divided into several groups. However, our method has an advantage in that it can treat the case in which subjects belong to groups which are not determined by discrete variables but by continuous ones. For the data of the latter kind to be analyzed by the method using equating, the non-grouped data have to be divided into several groups by arbitrary criteria, which means the loss of information, since subjects of similar but different values of covariates are treated as a single group

\section{Appendix}

The information matrix for the estimation Method II :

$$
\begin{aligned}
& E\left(\frac{-\partial^{2} \ln L}{\partial \boldsymbol{\beta}_{j} \partial \boldsymbol{\beta}_{j}^{\prime}}\right) \cong \sum_{q=1}^{K} \sum_{i=1}^{N}\left(\begin{array}{cc}
1 & \sigma_{i} \xi_{q}+\mu_{i} \\
\sigma_{i} \xi_{q}+\mu_{i} & \left(\sigma_{i} \xi_{q}+\mu_{i}\right)^{2}
\end{array}\right) P_{q j} Q_{q j} f\left(\xi_{q} \mid \boldsymbol{x}_{\boldsymbol{i}}, \boldsymbol{\beta}, \boldsymbol{\gamma}, \boldsymbol{\delta}\right), \\
& E\left(\frac{-\partial^{2} \ln L}{\partial \boldsymbol{\beta}_{\boldsymbol{k}} \partial \boldsymbol{\beta}_{j}^{\prime}}\right)=0,(k \neq j) \\
& E\left(\frac{-\partial^{2} \ln L}{\partial \boldsymbol{\gamma} \partial \boldsymbol{\beta}_{j}^{\prime}}\right) \cong \sum_{q=1}^{K} \sum_{i=1}^{N} \beta_{2 j} \frac{\partial \mu_{i}}{\partial \boldsymbol{\gamma}}\left(1, \sigma_{i} \xi_{q}+\mu_{i}\right) P_{q j} Q_{q j} f\left(\xi_{q} \mid \boldsymbol{x}_{i}, \boldsymbol{\beta}, \boldsymbol{\gamma}, \boldsymbol{\delta}\right) \\
& E\left(\frac{-\partial^{2} \ln L}{\partial \boldsymbol{\delta} \partial \boldsymbol{\beta}_{j}^{\prime}}\right) \cong \sum_{q=1}^{K} \sum_{i=1}^{N} \beta_{2 j} \xi_{q} \frac{\partial \sigma_{i}}{\partial \boldsymbol{\delta}}\left(1, \sigma_{i} \xi_{q}+\mu_{i}\right) P_{q j} Q_{q j} f\left(\xi_{q} \mid \boldsymbol{x}_{\boldsymbol{i}}, \boldsymbol{\beta}, \boldsymbol{\gamma}, \boldsymbol{\delta}\right), \\
& E\left(\frac{-\partial^{2} \ln L}{\partial \boldsymbol{\gamma} \partial \boldsymbol{\gamma}^{\prime}}\right) \cong \sum_{q=1}^{K} \sum_{j=1}^{p} \sum_{i=1}^{N} \beta_{2 j^{2}}{ }^{2} \frac{\partial \mu_{i}}{\partial \boldsymbol{\gamma}} \frac{\partial \mu_{i}}{\partial \boldsymbol{\gamma}^{\prime}} P_{q, j} Q_{q j} f\left(\xi_{q} \mid \boldsymbol{x}_{i}, \boldsymbol{\beta}, \boldsymbol{\gamma}, \boldsymbol{\delta}\right) \\
& E\left(\frac{-\partial^{2} \ln L}{\partial \boldsymbol{\delta} \partial \boldsymbol{\gamma}^{\prime}}\right) \cong \sum_{q=1}^{K} \sum_{j=1}^{p} \sum_{i=1}^{N} \beta_{2 j}{ }^{2} \xi_{q} \frac{\partial \sigma_{i}}{\partial \boldsymbol{\delta}} \frac{\partial \mu_{i}}{\partial \boldsymbol{\gamma}^{\prime}} P_{q j} Q_{q j} f\left(\xi_{q} \mid \boldsymbol{x}_{i}, \boldsymbol{\beta}, \boldsymbol{\gamma}, \boldsymbol{\delta}\right) \\
& E\left(\frac{-\partial^{2} \ln L}{\partial \boldsymbol{\delta} \partial \boldsymbol{\delta}^{\prime}}\right) \cong \sum_{q=1}^{K} \sum_{j=1}^{p} \sum_{i=1}^{N} \beta_{2 j}{ }^{2} \xi_{q}{ }^{2} \frac{\partial \sigma_{i}}{\partial \boldsymbol{\delta}} \frac{\partial \sigma_{i}}{\partial \boldsymbol{\delta}^{\prime}} P_{q j} Q_{q j} f\left(\xi_{q} \mid \boldsymbol{x}_{i}, \boldsymbol{\beta}, \boldsymbol{\gamma}, \boldsymbol{\delta}\right)
\end{aligned}
$$

\section{References}

Andersen, E.B. (1980). Comparing latent distributions. Psychometrika, 45, 121-134.

Andersen, E.B., \& Madson, M. (1977). Estimating the parameters of the latent population distribution. Psychometrika, 42, 357-374.

Bock, R.D., \& Aitkin, M. (1981). Marginal maximum likelihood estimation of item parameters: Application of an EM algorithm. Psychometrika, 46, 443-459. 
Bock, R.D., \& Jones, L.V. (1980, reprint). The measurement and prediction of judgment and choice. Chicago: International Educational Services, (Originally published in 1968).

Bock, R.D., \& Lieberman, M. (1970). Fitting a response model for $n$ dichotomously scored items. Psychometrika, 35, 179-197.

Dempster, A.P., Laird, N.M., \& Rubin, D.B. (1977). Maximum likelihood estimation from incomplete data via the EM algorithm (with discussion). Joumal of the Royal Statistical Society, Series $B, 39,1-38$.

Harwell, M.R., Baker, F.B., \& Zwarts, M. (1988). Item parameter estimation via marginal maximum likelihood and an EM algorithm: A didactic. Journal of Educational Statistics, 13, $243-271$.

Jöreskog, K.G. (1971). Simultaneous factor analysis in several populations. Psychometrika, 36, 409426 .

Louis, T.A. (1982). Finding the observed information matrix when using the EM algorithm. Journal of the Royal Statistical Society, Series B, 44, 226-233.

Mislevy, R.J. (1984). Estimating latent distributions. Psychometrika, 49, 359-381.

Mislevy, R.J. (1985). Estimation of latent group effects. Joumal of the American Statistical Association, 80, 993-997.

Mislevy, R.J. (1987). Exploiting auxiliary information about examinees in the estimation of item parameters. Applied Psychological Measurement, 11, 81-91.

Mislevy, R.J., \& Sheehan, K.M. (1989). Information matrices in latent-variable models. Joumal of Educational Statistics, 14, 335-3̄̄0.

Ogasawara, H. (1986). Maximum likelihood estimation of criteria for age adjustment in intelligence test. Railway Labor Science, 40, 37-53, (in Japanese).

Ogasawara, H. (1990). Covariance structure model when the factor means and the covariances are functions of the third variable. Japanese Psychological Research, 32, 19-25.

Reiser, M. (1983). An item response model for the estimation of demographic effects. Journal of Educational Statistics, 8, 165-186.

Rigdon, S.E., \& Tsutakawa, R.K. (1983). Parameter estimation in latent trait models. Psychometrika, 48, 567-574.

Sanathanan, L., \& Blumenthal, S. (1978). The logistic model and estimation of latent structure. Journal of the American Statistical Association, 73, 794-799.

Sörbom, D. (1974). A general method for studying differences in factor means and factor structures between groups. British Joumal of Mathematical and Statistical Psychology, 27, 229.239.

Tsutakawa, R.K. (1984). Estimation of two-parameter logistic item response curves. Joumal of Educational Statistics, 9, 263-276.

(Received September, 1994. Revised October, 1994) 